\title{
Humanités et SHS dans les écoles d'ingénieurs en France : une approche sociohistorique
}

\section{Catherine Roby}

\section{(2) OpenEdition \\ Journals}

Édition électronique

URL : http://journals.openedition.org/trema/3636

DOI : $10.4000 /$ trema.3636

ISSN : 2107-0997

Éditeur

Faculté d'Éducation de l'université de Montpellier

Édition imprimée

Date de publication : 1 décembre 2017

Pagination : 17-34

ISBN : 979-10-96627-03-5

ISSN : 1167-315X

Référence électronique

Catherine Roby, «Humanités et SHS dans les écoles d'ingénieurs en France : une approche

sociohistorique », Tréma [En ligne], 47 | 2017, mis en ligne le 01 février 2018, consulté le 05 mai 2019.

URL : http://journals.openedition.org/trema/3636 ; DOI : 10.4000/trema.3636

Ce document a été généré automatiquement le 5 mai 2019.

Trema 


\title{
Humanités et SHS dans les écoles d'ingénieurs en France : une approche sociohistorique
}

\author{
Catherine Roby
}

\section{Introduction}

1 Depuis l'Ancien Régime, nombre d'écoles d'ingénieurs forment des professionnels à haut niveau de qualification destinés à évoluer dans des carrières qui les placent souvent proches des sphères du pouvoir décisionnel, dans les organisations de travail. C'est pourquoi, elles ont toujours veillé à procurer à leurs diplômés des apports disciplinaires élargis, et dépassant ceux des spécialités de sciences et techniques. Ainsi, de nombreuses recherches soulignent la présence des humanités, puis de l'économie, du droit et de la philosophie dans les curricula des écoles, puis progressivement et plus généralement des sciences humaines et sociales (SHS) (voir entre autres, Belhoste, 2003 ; Day, 1987/1991; Derouet et Paye, 2010 ; Didier, 2008 ; Dufour, 1998 ; Grelon, 1995 ; Lemaître, 2003 ; Picon, 1992).

Dans les premières écoles d'ingénieurs, les humanités étaient destinées à asseoir la position des élites et avaient donc un rôle de légitimation sociale (Belhoste, 2003 ; Picon, 1992). Après la Révolution française, le pouvoir technocratique mis en place, s'est appuyé sur la légitimité des savoirs scientifiques (Thoenig, 1987). Or, il ne semble pas exister de travaux qui se soient intéressés à une approche croisée de cette double légitimation (sociale et technocratique) des activités des ingénieurs. Les conséquences, dans leurs formations, de l'opposition entre les différentes formes de savoirs développés sur l'humain et le social, de la seconde moitié du XIX siècle à la première moitié du XXe siècle, ne semblent pas non plus avoir été étudiées. Par ailleurs, peu de travaux traitent de la relative absence, dans les formations d'ingénieurs, des savoirs développés depuis une quarantaine d'années par les SHS sur le rapport que l'humain entretient à la science et à la technique. De la même façon, la faible prise en compte réflexive dans les 
formations, des réalités de contexte des activités d'ingénierie, de la standardisation des modèles d'organisation et des discours sur la rationalité du travail, semble guère questionnée (Roby, 2014).

3 Ainsi, malgré les enjeux des activités d'ingénierie pour nos sociétés technologiques occidentales, les tensions, liées à l'introduction et aux finalités des humanités et des SHS dans les formations d'ingénieurs, engendrent depuis toujours un caractère problématique du positionnement de ces disciplines, quelle que soit la diversité des écoles (Albero et Roby, 2014), et cette situation reste peu étudiée.

4 En prenant appui sur une approche sociohistorique, tant des formations que du contexte conflictuel de la naissance des sciences sociales, cette contribution ${ }^{1}$ vise alors à éclairer les difficultés récurrentes du positionnement des humanités et des SHS dans les écoles d'ingénieurs. Cette double perspective permet de mettre en évidence la persistance de l'élitisme, l'existence de différentes cultures techno-scientifiques des ingénieurs et de leurs formations, ainsi que le rapport spécifique à la science et aux savoirs sur l'humain et le social que les ingénieurs ont développé.

5 La première partie présente la diversité des ingénieurs et de leurs formations marquées par un élitisme, depuis les services qu'ils ont rendus à la royauté puis leur mise à disposition de l'État. Ces « honnêtes hommes » exerçaient dans des corps sélectifs dont les normes et les valeurs ont été conservées à travers le temps. Si les formations d'ingénieurs se sont progressivement ouvertes, elles n'en ont pas moins gardé leur caractère sélectif et plus ou moins élitiste. Au-delà d'une certaine forme d'unité, les écoles d'ingénieurs témoignent d'une diversité de cultures technoscientifiques, liées notamment à leur sociohistoire, aux spécialisations disciplinaires et aux spécificités des secteurs d'activité.

6 La seconde partie porte sur les savoirs que les ingénieurs ont développés sur l'humain et le social au moment même où les SHS s'institutionnalisaient. La sociologie développée par Le Play à l'École des mines s'inscrit dans le catholicisme ambiant; elle conduit à l'affirmation du rôle social de l'ingénieur, garant de la paix sociale, dans une période troublée par les bouleversements économiques, politiques, religieux et sociaux. Dans ce contexte, la sociologie durkheimienne, laïque et républicaine, s'oppose à la sociologie leplaysienne, catholique et utilitaire.

7 La troisième partie aborde les opportunités et les difficultés de positionnement des humanités, puis des SHS, dans les écoles d'ingénieurs. Si les humanités ont eu pour principale fonction de légitimer la position sociale des ingénieurs, comme dans toute formation de la classe dirigeante, l'approche des réalités économiques, politiques et juridiques a également fait partie de leur formation. Ensuite, alors qu'ils se revendiquaient un rôle social, la formation humaine et sociale des ingénieurs s'est progressivement développée au XXe siècle, jusqu'à ce que la concurrence des écoles de commerce, dans les années 1970, impose la formation au management ; si cette formation reste centrale dans les écoles d'ingénieurs, elle ne s'appuie pas nécessairement sur les savoirs académiques construits par les SHS.

\section{Les écoles d'ingénieurs : entre identité commune et pluralité}

8 Depuis l'Ancien Régime, les écoles d'ingénieurs ont développé un modèle de formation élitiste, destiné à préparer de futurs professionnels, mis successivement au service des 
plus hautes fonctions du royaume, de l'État et des entreprises industrielles. Au fil du temps, les écoles, dans la diversité de leurs cultures technoscientifiques, ont conservé ce modèle bien qu'elles se soient ouvertes dans leurs modalités de recrutement, et que leurs débouchés professionnels aient parfois perdu de leur prestige.

\section{1. Aux origines, un modèle élitiste}

Les corps des ingénieurs ont été créés dans le prolongement des corporations de l'Ancien Régime; les ingénieurs du Roi sont ainsi passés au service de la puissance publique. Au XIXe siècle, les corps techniques de l'État ont mis leur puissance au service du bien collectif de la nation. Les ingénieurs de ces corps constituaient une élite, «c'est à dire un ensemble humain socialement distinct et situé en dessus du reste de la société » (Thoenig, 1987, p. 14). En France, les ingénieurs ont ainsi acquis, dès le XVIIIe siècle, la ferme conviction d'appartenir à une élite courageuse et détentrice du savoir, pour le bien commun (Picon, 2007). Plus tard, le service de l'État, c'est-à-dire de l'intérêt général, s'est manifesté comme une essence, un attribut des ingénieurs des corps d'État (Gervais, 2007). Tout était prévu dans leur formation pour façonner des élites complètes, lettrées et cultivées, c'est à dire pour faire d'eux d'honnêtes gens capables de tenir leur rang social, de rendre honneur à leur corps d'appartenance, par l'adoption de valeurs et de comportements de référence (Belhoste, 2003 ; Picon, 1992). Ces normes et valeurs se sont transmises aux ingénieurs civils à la création de l'École Centrale (1829); elles sont le ciment de la construction d'une solidarité entre les membres des écoles d'ingénieurs, qui dans leur diversité croissante, se sont toutes et toujours caractérisées par leur sélectivité, quelle qu'en soit la forme.

10 Considérée comme une originalité du modèle français de formation des ingénieurs, il s'agit là en fait de la conservation du stéréotype d'excellence (Giré, Béraud et Déchamps, 2000) sur lequel reposent d'ailleurs les pratiques de bizutage et d'intégration ; adoptées par la plupart des écoles, elles inaugurent l'entrée dans un cercle fermé (Corbières, 2003).

11 De plus, les conditions de travail et de vie, dans les écoles d'ingénieurs, incitent à une certaine autarcie, dans un entre soi, d'ailleurs longtemps resté « masculin » et peu ouvert aux étrangers (Veltz, 2007); elles développent un sens de l'exception. Basé sur le modèle traditionnel et socialement élitiste de l'Ancien Régime, toute l'organisation des écoles vise toujours à créer un espace de sociabilité des élèves, qui les maintient dans un univers clos, spatialement et mentalement, afin qu'ils intègrent au mieux les valeurs qu'on leur inculque (Corbières, 2003 ; Lemaître, 2007 ; Picon, 1992).

Le caractère élitiste de ces écoles apparait également dans la pérennité de certains rites, comme les concours et les cérémonies de remise des diplômes par exemple, des actes d'autorité symbolique, qui posent et légitiment une distinction sociale. Ces rites d'institution sont performatifs, les personnes distinguées se reconnaissent et sont reconnues comme naturellement distinctes et se comportent comme telles (Belhoste, 2003).

13 L'histoire des formations d'ingénieurs en France s'est donc d'emblée inscrite dans une forme élitiste, offrant autant un statut et une distinction sociale que des compétences scientifiques et techniques. Bien que ces formations se soient démocratisées au fil du temps, elles n'ont néanmoins pas perdu toute forme d'élitisme. 


\section{2. Un élitisme maintenu dans la diversité des écoles et des voies de formation} initiale. Malgré la mise en place en 1974 d'une filière de formation continue et la création en 1990 des formations en partenariat, la réalité reste marquée par l'élitisme des écoles. Le titre distinctif des diplômés de certaines formations par apprentissage : «Ingénieur des techniques de l'industrie », tout comme la lenteur de la mise en place dans les écoles des diplômes par validation des acquis de l'expérience, témoignent de la force de ce trait identitaire.

\section{3. Les cultures technoscientifiques des ingénieurs et de leurs formations}

17 Bien qu'une «mythologie de la liberté d'entreprendre » ait pu assurer au début du XXe siècle l'unité des ingénieurs, en les rapprochant par-delà leurs différences de statut et de formation (Day, 1987/1991), il n'y ni profession ni représentation unifiée de l'ingénieur. Ce sont des dynamiques historiques, celles de leurs modes de formation, qui donnent sens à la construction identitaire des ingénieurs (Roquet, 2005). En effet, les écoles diffèrent selon leur âge, leur contexte de création, leurs modes de recrutements, leur tutelle, les orientations de spécialisation, les domaines de professionnalisation. Les ingénieurs et leurs formations constituent donc des mondes pluriels.

Les écarts observés selon les secteurs d'activité, métallurgie versus chimie ou électronique par exemple (Crawford, 1984 ; Lasserre, 1989), dans le fonctionnement des entreprises et le positionnement professionnel des ingénieurs, peuvent être mis en relation avec le rôle inégal qu'ont joué les ingénieurs dans la diffusion de la rationalisation, selon les secteurs d'activité, dans le premier tiers du $\mathrm{XX}^{\mathrm{e}}$ siècle.

19 En effet, l'organisation scientifique du travail (OST) et le système fordien avaient pour but d'assurer la productivité par la continuité du flux de production. Mais certains secteurs, comme ceux de la chimie, de l'électronique ou encore ceux des mines et du bâtiment, 
pour différentes raisons, n'avaient pas l'emploi des modes d'organisation du travail de l'OST (Moutet, 1997).

Par ailleurs, la pluralité des politiques identitaires des ingénieurs est en relation avec la diversité des représentations épistémologiques des savoirs scientifiques qu'ils utilisent et les valeurs véhiculées par ces savoirs dans les différents domaines professionnels (Downey et Lucena, 2004). Ainsi, dans une école qui offre des formations dans des spécialités multiples, les écarts identitaires peuvent être importants (Fraysse, 1998, 2000, 2006 ; Giré et al., 2000).

21 Les secteurs d'activité, et les spécialisations disciplinaires et techniques associées, s'avèrent donc des critères de différenciation identitaire des ingénieurs, susceptibles d'influencer les comportements, les pratiques et les valeurs de ces derniers. Il semble, par conséquent, que la notion de "culture technoscientifique » des formations d'ingénieurs puisse permettre d'envisager l'institutionnalisation des façons de faire et de penser de leurs membres, à travers «les processus d'apprentissage, de routinisation ou de changement dans la longue durée » (Godelier, 2009), comme c'est le cas pour la culture d'entreprise.

En effet, à partir de la notion de "culture scientifique et technique » définie par Godin, Gingras et Bourneuf (1998) comme «l'expression de l'ensemble des modes par lesquels une société s'approprie la science et la technologie», on peut décliner, pour les communautés des écoles d'ingénieurs, la notion de «culture technoscientifique ». Les exigences du travail dans un domaine particulier influent sur la manière de formuler et de résoudre les problèmes (Scribner, 1983, citée par Bruner, 1996/2008); le travail, ou plus généralement une praxis, constitue le prototype d'une culture. Dans une certaine mesure, il semble donc possible de considérer que la formation des écoles d'ingénieurs conduise, par les modes de socialisation qu'elle propose, à des façons d'envisager le monde, à l'intériorisation de modes de pensée, d'action et de réflexion (Garçon, 2004 ; Giré et al., 2000 ; Lemaître, 2007).

\section{Les ingénieurs et les savoirs sur l'humain et le social}

La sociologie française présente la caractéristique d'une double naissance dans la seconde moitié du XIXe siècle. Deux approches s'opposent dans un contexte conflictuel; l'une, développée par un ingénieur des mines, Frédéric Le Play, et l'autre, par un philosophe, Emile Durkheim. À la fin du XIXe siècle, la science industrielle, initialement fondée par les ingénieurs centraliens pour le perfectionnement de l'industrie, est réinvestie sous la figure de l'OST. Avec l'aura de la rationalité de la science, elle se présente également comme une science du social.

\section{1. Un ingénieur parmi les fondateurs de la sociologie}

Frédéric Le Play (1806-1882) a bâti et développé à l'École des mines de Paris une « science sociale des ingénieurs », immédiatement effacée par la « science sociale » des philosophes (Durkheim, Fouillée) et des juristes (Tarde, Worms), élaborée dans d'autres cadres de connaissance (Le Play, Savoye et Audrain, 2008). 

d'études monographiques de familles ouvrières, appréhendées par leur budget. L'exactitude de la réalité comptable et le constat neutre de la vie de ces familles devaient garantir la rigueur scientifique (David, 2003 ; Savoye, 1995). Avec ses principes d'étude du commandement, liés à la famille, l'ordre et la hiérarchie, la sociologie leplaysienne a été particulièrement bien accueillie dans les milieux dirigeants, de catholiques et d'ingénieurs, qui craignaient le socialisme.

En effet, par ses capacités d'analyse et d'intégration des dimensions économiques et sociales, de prise en compte de la vie ouvrière dans l'exercice de ses fonctions, l'ingénieur assure un rôle social ; il devient donc un ingénieur social, à même d'assurer la paix sociale, dans l'esprit du catholicisme social (Savoye, 1994). Dans un contexte de violentes manifestations ouvrières et à une période dans laquelle les élites de la société française se laissent submerger par la crainte des affrontements, des foules dangereuses et imprévisibles, des ouvriers et du socialisme, la paix sociale est l'idéal rêvé (Cohen, 2003; Segrestin, 1996). Les connaissances techniques des ingénieurs sont mobilisées au nom du bien de tous, elles garantissent leur rôle d'intermédiaire, comme experts neutres, entre les patrons et les ouvriers (Grelon, 1984, 1986). La figure de l'ingénieur social est portée par des personnalités exerçant leurs fonctions dans la plupart des grandes écoles d'ingénieurs (Kalaora et Savoye, 1992 ; Savoye, 2008). Elle sera diffusée jusque dans les années 1930, permettant alors aux ingénieurs de défendre leur place, en même temps que leur titre, en période de crise (Cohen, 2003, 2011; Grelon, 1986,1995). des contextes, sociaux et politiques, antagonistes, qui éclairent les relations instaurées entre les ingénieurs et les savoirs sur l'humain et le social, aux débuts même de l'institutionnalisation des nouvelles branches de savoirs disciplinaires que constituent les SHS.

\section{2. Le contexte conflictuel de la naissance des sciences sociales}

C'est pour tenter de résoudre les problèmes induits par les bouleversements sociaux, économiques et politiques, nés de la rapidité des progrès techniques, de l'industrialisation et de l'urbanisation, que s'est développé le catholicisme social, au cours des années 1830 et 1840. À la fin du XIXe siècle, après les violentes secousses d'une crise économique en Europe, et dans un contexte favorable de reconfiguration des disciplines scientifiques, une pensée scientifique du social s'est installée. Les sciences sociales nourrissaient alors l'espoir de fournir les outils du diagnostic et de la prévention, en s'intéressant aux questions des conditions de travail et de vie des masses laborieuses, d'habitat, d'hygiène, et de pauvreté. Ainsi se mêlent dans les débats sur l'amélioration de l'avenir, les questions sociales, syndicales, politiques, idéologiques, et celles des sciences du social, dans un voisinage périlleux (Boure, 2007 ; Cohen et Baudouï, 1995; Pomian, 2010).

L'œuvre de Durkheim, illustrant la fonction politique de la science (Lenoir, 2004), s'inscrit dans le contexte du XIXe siècle, où science sociale rime avec social et socialisme (Blanckaert, 2006; Dortier, 2006) ; elle effraie donc le pouvoir politique comme tous les pouvoirs en place. La sociologie universitaire de Durkheim, science d'une morale laïque et républicaine, a remis en cause les fondements même du catholicisme, elle est donc apparue comme un danger pour l'Église (Serry, 2004). En montrant que les catégories de 
la pensée sont des constructions sociales, elle a refusé de naturaliser le social. En minimisant aussi le rôle des élites individuelles dans l'histoire, elle est allée à l'encontre de la vision élitaire du catholicisme et des conservateurs de droite, dont étaient majoritairement issus les ingénieurs de l'époque. Cela lui a valu d'être assimilée à un matérialisme négateur de l'individu. Son rejet est devenu unanime chez les intellectuels catholiques qui ont alors élaboré leur propre sociologie, conforme aux enseignements de l'Église, la sociologie catholique, inspirée de la sociologie leplaysienne (ibid.). Cette sociologie s'est inscrite dans le combat qui a opposé les républicains laïques et les partisans du catholicisme monarchique pour le contrôle du pouvoir politique. Le type d'expertise des enquêtes leplaysiennes, crédible et argumenté, a permis de montrer que sciences sociales et catholicisme ne s'opposaient pas et n'entraient pas en conflit. L'Église a ainsi pu montrer qu'elle avait les capacités de combattre la misère du prolétariat, et la sociologie catholique leplaysienne est alors apparue comme un système de pensée à imposer (Heilbron, Lenoir et Sapiro, 2004 ; Lenoir, 2004).

Ainsi, dans la première moitié du XXe siècle, ingénieurs sociaux et hommes de lettres ont lutté pour garder le monopole de leurs discours sur le monde social, face à la sociologie naissante, en voie d'institutionnalisation (Boltanski, 1981; Henry, 2004, 2006). Les sciences sociales ont donc dû s'émanciper des tentatives de captation, tant des pouvoirs religieux que politiques ou économiques, pour conquérir une relative autonomie (Heilbron et al., 2004). Elles n'étaient pas compatibles avec la vision élitaire des ingénieurs.

31 Au milieu de ces conflits, et peut-être pour les dépasser, l'organisation scientifique du travail (OST) a permis de revisiter à nouveaux frais la science industrielle, à l'origine de la création en 1829 de l'École Centrale destinée à former des ingénieurs généralistes.

\section{3. Une science industrielle globalisante pour former les centraliens}

32 À la création de l'École Centrale, l'idée de science industrielle s'est concrétisée en opposition au clivage entre les théories scientifiques et leurs applications industrielles. Elle était envisagée comme une réponse à des attentes concernant la représentation cohérente, unifiée et homogène d'une industrie émergente, dont les responsables souhaitaient qu'elle soit considérée comme objet de science, afin de lui assurer un développement autonome. La science en question devant être le fondement théorique de l'École centrale, et l'outil de perfectionnement de l'industrie, elle devait être une activité réservée à ses ingénieurs, auxquels elle devait fournir mode de pensée et guide d'action. Il s'agissait de leur donner une légitimité, celle de la technocratie, pour agir de façon universelle dans l'entreprise (Grelon, 1996). En effet, après la Révolution française de 1789, la technocratie, à sa naissance, s'était appuyée sur les sciences, considérées comme seule source de rationalité technique pour instaurer une double domination, celle des savoirs formels sur les connaissances issues de la pratique, et celle des hommes formés dans les écoles sur les hommes formés par l'expérience du métier. Dans cette représentation de la raison, entièrement fondée sur l'application et la neutralité des sciences, les théories affranchissent des perceptions humaines de la pratique (Belhoste, 2003).

33 Au tournant du XXe siècle, la rationalisation que propose l'OST présentée comme nouvelle science industrielle par Henry Le Chatelier ${ }^{2}$ est parée de l'objectivité de la science. 
Le Chatelier est certainement la figure la plus emblématique d'un culte de la rationalisation ${ }^{3}$ et de la défense d'une science industrielle, véritable théorie de l'action, mobilisant les critères rationnels de la science pour ses applications industrielles. Les ingénieurs étaient favorables aux méthodes de Taylor, et parfois de plus vifs promoteurs de l'OST que ne l'était le patronat. Ils voyaient, dans cette organisation, une possibilité d'asseoir leur pouvoir technique; cela nécessitait une légitimation par la science pour gagner une position dominante incontestable, directement liée au pouvoir disciplinaire qui avait accompagné le développement industriel depuis la fin du XVIIe siècle (Grelon, 1986 ; Henry, 2012 ; Letté, 2004). Cette position implique l'extension de la légitimité scientifique sur le contrôle de la sphère industrielle et sociale. Elle sous-tend un projet de rationalisation et de légitimation d'un État technocratique, avec un gouvernement par les scientifiques et ingénieurs, alors même que c'est l'efficacité du savoir qui prime sur les fondements théoriques de ce savoir dans la nouvelle science industrielle (Garçon, 2004 ; Henry, 2012 ; Letté, 2010 ; Picon, 1992 ; Picon et Chatzis, 1992).

Pour Henry Le Chatelier, la science industrielle, présentant les lois de l'ordre social comme des lois naturelles et universelles, était à construire comme une science de l'homme et devait être enseignée dans les formations d'ingénieurs où elle était légitimée par le positivisme ambiant de la fin du XIXe et du début du XXe siècles. Elle rendait inopérante la sociologie durkheimienne, d'autant plus difficile à accepter que Le Chatelier faisait partie des milieux de la droite conservatrice, et qu'il était lié aux milieux industriels, catholiques et conservateurs (Eidelman, 1986 ; Garçon, 2004 ; Henry, 2012 ; Letté, 2004).

Dans les écoles d'ingénieurs, ces modes de penser et d'agir se sont diffusés progressivement, assurant la rationalisation des systèmes de production industrielle, et visant toujours l'amélioration de la productivité. La rationalisation du travail, telle que la propose l'OST, doit rendre le travail moins pénible, donc plus humain. Les ingénieurs sont les principaux acteurs de la rationalisation qu'ils se donnent pour mission d'étendre du domaine de la production industrielle aux autres domaines de la vie sociale (Letté, 2010). C'est ce qui les a sans doute rendus peu enclins à s'intéresser aux sciences sociales naissantes, dans un contexte social et politique difficile. Cette situation a probablement déterminé un rapport particulier à l'humain et au social dans les différentes écoles d'ingénieurs, inscrites dans un ordre social établi qu'elles entretiennent en formant une élite rompue à la rationalité des sciences de la nature et de la technique.

\section{Des humanités aux SHS dans la formation des ingénieurs}

Dans le cadre de la formation élitiste des ingénieurs, les humanités ont servi à asseoir leur position sociale. Tel était d'ailleurs leur rôle dans toutes les formations de la classe dirigeante. Mais parallèlement, les fonctions marchandes de l'ingénieur ont nécessité une formation aux réalités économiques, politiques et juridiques. Progressivement, au XXe siècle, s'est manifesté le besoin d'une formation humaine et sociale des ingénieurs, basée sur le commandement des hommes, pour qu'ils puissent assurer le rôle social dont ils se revendiquaient. Dans les années 1970, face à la concurrence des écoles de commerce, la formation au management s'est imposée; si elle reste centrale dans les écoles d'ingénieurs, elle ne mobilise pas de façon systématique les savoirs académiques développés par les SHS. 


\section{1. Des humanités pour former l'élite} procurer, aux futures élites de la Nation, l'acquisition d'une culture générale nécessaire, issue des héritages savants classiques, et supposée comme «le préalable à une action juste et efficace au service du progrès » (Lemaître, 2007). Au XIX siècle, l'enseignement des humanités, par l'étude des langues anciennes et textes classiques, est intégré à la formation traditionnelle des élites, qu'elles soient celles de la haute fonction publique, des corps d'ingénieurs de l'État ou des professions libérales (Day, 1987/1991). La formation classique n'a pas pour unique but la rédaction de rapports ou l'acquisition de savoirs spéciaux, il s'agit aussi de partager une langue de culture. Dans les écoles d'ingénieurs, les humanités devaient donc permettre aux futures élites de tenir leur rang social par la maîtrise des codes linguistiques de la classe dirigeante, en particulier pour la langue écrite. La formation littéraire par les humanités était donc au fondement du façonnage des élites complètes, en raison des capacités discursives qu'elles conféraient (Belhoste, 2003 ; Letté, 2004 ; Picon, 1992).

En accolant le stigmate de dilettantisme à la culture générale, identifiée alors aux humanités qui formaient les classes dirigeantes, et en développant la sociologie en France, dans les facultés de lettres, Durkheim affirmait ainsi la place de la sociologie contre la culture humaniste. Les lettrés, dont cette culture formait le capital symbolique, ont violemment réagi. La barrière scolaire des humanités classiques et du latin était en fait une barrière sociale. L'éducation littéraire et les humanités classiques assuraient aux futures élites une aisance en société, les préparant ainsi à intégrer les classes dirigeantes et les rassurant quant à leur supériorité intellectuelle (Heilbron, 2006; Henry, 2000; Sapiro, 2004).

$39 \mathrm{Au}$ cours du XXe siècle, l'enseignement des humanités s'est toujours répandu en priorité dans des écoles prônant une formation généraliste, dans l'idée d'une hiérarchisation des écoles et dans une volonté de démarcation des formations d'ingénieurs de spécialité technique. Ces projets de formation, dans une dimension culturelle et humaniste, sont mis en avant pour marquer l'appartenance à des professions socialement prestigieuses (Derouet et Paye, 2010).

Mais parallèlement à l'enseignement des humanités dans la formation des premiers ingénieurs, l'activité de ces derniers s'était calée sur le mouvement de la production marchande, et le développement économique avait promu le thème de l'utilité au rang des réflexions sociales majeures. La capacité des ingénieurs des ponts et chaussées à prendre en compte les réalités économiques, sachant concilier la qualité de leurs ouvrages, destinés aux services de l'État, et adaptés aux exigences de durabilité, aux contraintes économiques et sociales, a fait durablement leur succès. Les enseignements juridiques, d'économie politique et industrielle, ont aussi fait partie de la formation des ingénieurs dès le XIX ${ }^{\mathrm{e}}$ siècle, que ce soient les polytechniciens, ceux des grands corps de l'État ou les ingénieurs civils (Belhoste, 2003 ; Derouet, 2013 ; Picon, 1992).

41 Puis, au XXe siècle, c'est vers la direction des hommes et le management des entreprises que se sont désormais orientés les enseignements dits complémentaires des sciences et des techniques dans les formations d'ingénieurs. 


\section{2. Apprendre à manager et à communiquer : la formation humaine et sociale}

42 Au XXe siècle, dans les années 1930, la dimension humaniste et sociale des formations d'ingénieurs s'affirme et devient fortement valorisée dans une période de crise pendant laquelle se réaffirme le rôle social de l'ingénieur (Grelon, 1986; Moutet, 1995; Remoussenard, 2006; Robert, 1986). En cette même période, la Fondation Rockfeller promeut, sur le modèle américain, le développement de sciences sociales empiriques et appliquées, pour produire des techniques et des outils de contrôle du monde social. Elle a aussi pour but de s'opposer à la tradition critique des sciences sociales, issue du marxisme (Boltanski, 1981 ; Henry, 2004).

En 1937, très attachée à la fonction sociale de l'ingénieur, dans le cadre de la doctrine sociale de l'Église, la puissante Union des syndicats d'ingénieurs catholiques (USIC) organise des conférences de formation sociale. Dans ces années, se développe donc l'idée d'une préparation morale des ingénieurs, par une formation sociale devant faire d'eux des chefs destinés à commander les ouvriers et à exercer sur eux une influence exemplaire (Moutet, 1997). Le commandement des hommes au travail est donc la principale préoccupation pour la formation humaine et sociale des ingénieurs, dans le contexte de la rationalisation du travail où le caractère de l'ingénieur est une valeur primordiale.

Le caractère nécessaire ou indispensable des chefs et l'idée de la formation des chefs imprègne fortement et durablement les milieux industriels et ceux des ingénieurs (Crawford, 1984; Laot, 1999). À la fin du XX siècle, Monnet (2013) évoque les cadres dirigeants de Renault comme une " étonnante tribu, affairée à spécifier minutieusement les parures du pouvoir des chefs et veillant à l'entretien méticuleux de la voie hiérarchique » (ibid., p. 21). En 2009, «former des chefs » était encore présenté par la CGE ${ }^{4}$ comme un défi pour les dispositifs de formation d'ingénieurs.

45 C'est après la seconde guerre mondiale que se sont amplifiée l'ouverture et la diversification des formations d'ingénieurs. La question de la formation humaine et sociale des ingénieurs a alors été réinterrogée, le patronat souhaitant qu'ils aient une meilleure connaissance économique des entreprises et sachent mieux communiquer pour éviter les conflits (Derouet et Paye, 2010). Puis, à partir du début des années 1970, le patronat comprend qu'il faut un nouveau mode de commandement, différent de celui de l'élan de la rationalisation technique (Moutet, 1997). En outre, avec le développement et la concurrence des écoles de commerce, la légitimité de l'ingénieur à gérer ces nouveaux pouvoirs est limitée par son image de " technicien ». C'est pourquoi il doit «accepter de nouveaux devoirs, comme celui de la culture générale de l'ingénieur pour communiquer et interpréter l'environnement de l'entreprise " (Decomps cité par Grelon et Marry, 1996). Cette culture générale englobe le droit du travail, l'économie, la gestion et les langues étrangères. Dans ces années 1970, le Centre national du patronat français (CNPF) a donc imposé la légitimité d'une formation au management dans les écoles d'ingénieurs (Derouet et Paye, 2010).

Aujourd'hui, c'est encore et toujours au nom de son intérêt pour les fonctions managériales qu'est présentée la formation humaine et éthique des ingénieurs (Canepa, Folz et Blazy, 2009). Si cette formation reste centrale dans les préoccupations des acteurs des écoles d'ingénieurs, elle n'intègre pas nécessairement de façon explicite les travaux de recherche afférents en SHS (Roby, 2014). 


\section{3. Une diffusion partielle des SHS dans les formations d'ingénieurs}

47 Au début des années 1950, Friedman (1952), l'un des pionniers de la sociologie du travail, pointe les transformations sociales qu'engendrent les savoirs techniques, il insiste sur la nécessité d'une réflexion sociale dans tout enseignement technique. En considérant l'évolution technique comme une évolution humaine, il ne s'agit plus d'en accepter les conséquences, mais de développer un esprit critique pour éclairer les possibilités et le sens de nos choix et décisions.

Aider les hommes à prendre conscience de leur condition, et augmenter leurs chances de liberté concrète : tels sont les buts d'une connaissance des SHS.

Ces propos apparaissent à un moment de mise en cause d'une technique que des hommes ne développeraient plus que pour elle-même, oubliant de la mettre au service de leurs semblables (Ellul, 1954). Dans ce contexte, une étude des programmes des écoles d'ingénieurs indique la grande faiblesse, voire encore parfois l'absence totale de formation administrative, économique et sociale. Par la suite, dans les années 1950 et 1960, les écoles se sont interrogées sur l'intérêt des SHS pour les formations, mais sauf exception, elles ont choisi de leur réserver une place marginale (Dufour, 1998). En effet, dans les écoles d'ingénieurs, les aptitudes relationnelles, apparues comme une nécessité du renouvellement des modes d'encadrement et de management, ont en fait plus souvent été appréhendées dans les cours de communication que par l'enseignement de la psychologie et de la sociologie (Brucy, 1999 ; Tanguy, 2001). En outre, à l'époque, la formation humaine et sociale des ingénieurs est considérée comme relevant principalement du ressort de l'expérience professionnelle, bien plus que de la formation initiale des écoles (Dufour, 1998, Remoussenard, 2006).

Mais en 1953, dans la mouvance du plan Marshall, le Conseil économique insiste sur la nécessité d'introduire les réalités économiques et humaines du monde industriel dans la formation des ingénieurs et des cadres techniques. En pointant les lacunes des formations initiales sur ces aspects, il propose de substituer à quelques programmes de culture générale des notions relatives aux sciences économiques et sociales ainsi qu'aux relations humaines (Remoussenard, 2006). Ainsi, à partir du milieu des années 1950, l'influence américaine a amené progressivement l'idée d'une spécialisation en SHS (psychologie, sociologie, économie politique) (Tanguy, 2001), mais c'est surtout à partir des années 1960 et 1970 que les SHS (autres que le droit et l'économie administrative, politique ou industrielle, disciplines déjà présentes dans certaines écoles) ont été introduites plus généralement dans quelques formations d'ingénieurs.

51 Cependant, s'il est admis à la fin des années 1970 que les SHS peuvent contribuer à la formation des ingénieurs, les questions sur la nature précise de cette contribution demeurent. En 1982, l'UNESCO publie un rapport pointant la nécessité d'élaborer un processus de formation des ingénieurs, qui puisse permettre une connaissance approfondie relevant des SHS, associée à la technique, pour résoudre les problèmes complexes d'environnement (Brancher, 1982). 


\section{4. Des SHS pour affronter la complexité et l'incertitude?}

52 Si les SHS sont si sollicitées dans les formations d'ingénieurs, c'est en grande partie en raison de leur potentialité d'aide à l'apprentissage et au développement des aptitudes interprétatives, à l'enquête et au dialogue. Mais les élèves-ingénieurs, dans leur globalité, ne sont pas familiers des SHS, en raison de leurs parcours scolaires très tôt orientés par les mathématiques, les sciences physiques et les sciences de l'ingénieur. La compréhension en profondeur et le type de questionnement qui la guide ne correspondent pas aux capacités qu'ils ont développées, des savoir-faire procéduraux, une assimilation rapide, l'obsession de résultats absolus. Bien qu'elles ne s'y opposent pas a priori, les démarches herméneutiques des SHS ne sont pas si faciles à adopter pour eux ; elles les mettent mal à l'aise (Dufour, 1998 ; Lemaître 2003 ; Minguet, 2001). Leur curiosité peut les pousser à s'intéresser à ces disciplines, mais l'importance des écarts épistémologiques entre sciences de l'ingénieur et SHS freinent souvent ce mouvement, bien qu'il existe des écarts selon les cultures technoscientifiques des écoles (Roby, 2014). Il n'est pas toujours facile aux élèves-ingénieurs, habitués à la rapidité d'obtention de résultats dans leur résolution de problèmes, de se familiariser avec des SHS qui ne proposent que des résultats partiels et relatifs, même si dans le cadre d'une explication contextualisée, elles peuvent parfois faire l'objet d'une certaine généralisation. Cependant, elles n'ont pas de lois universelles à proposer, elles n'ont pas à transmettre de vérités indiscutables. Elles sont de plus traversées par une diversité de courants théoriques, tout en mobilisant des méthodologies variées.

Les sciences de l'ingénieur privilégient des formes de rationalité et de modélisation des problèmes hors de tout contexte humain et social ; elles ne préparent pas les élèvesingénieurs à affronter la complexité mouvante des situations réelles. D'où les difficultés, souvent soulignées au sujet des approches réductionnistes, face à des problèmes managériaux. Ceux-ci sont vus d'emblée comme un défaut de procédure auquel il faudrait remédier par une action corrective, alors même que des approches herméneutiques viseraient la compréhension personnelle d'une situation complexe, impliquant des facteurs organisationnels et humains, économiques, en préalable à l'action (Dufour, 1998 ; Lemaître 2003 ; Minguet, 2001). Mais ce type d'approche est jugé trop peu rationnel par des ingénieurs qui se méfient comme de la peste de l'irrationnel et qui ont «choisi leur formation pour ne pas y toucher $»^{5}$. La rationalité est en effet souvent jugée caractéristique des ingénieurs, manifestée par leur besoin de raisonner de façon inductive et structurée sur des faits appréhendés comme solides et durs. Il leur est difficile d'envisager différentes formes de rationalité et d'intelligence; leur capacité à apprécier les savoirs constitués par des sciences interprétatives (toujours partiels et situés dans l'espace et le temps), ainsi qu'à percevoir le monde social comme intrinsèquement symbolique, ou la vérité comme mouvante, demeure problématique.

Si les disciplines de SHS font l'objet d'un certain dédain de la part des élèves-ingénieurs (Didier, 2008), ce n'est donc pas seulement en raison de la faible valeur qu'elles donnent à leur diplôme sur le marché du travail (Dab, 2008). Le manque de motivation pour des enseignements en SHS, considérés comme difficiles ou inutiles pour des étudiants peu réceptifs, est avéré, surtout en l'absence de vécu professionnel significatif (AERES, 2010; Lourdel, 2005). Dans le contexte des écoles d'ingénieurs, où prévalent le rationalisme et la croyance dans le progrès par la technique, des disciplines comme la sociologie, par 
exemple, qui montre «la multiplicité des acteurs, qui révèle la structure des rapports sociaux et des pouvoirs, risque de déstabiliser. Elle n'a pas d'utilité pratique pour l'ingénieur, car elle va à l'encontre d'idéaux qui servent de guide pour l'action » (Dufour, 1998). Cette perception perdure manifestement dans les formations (Roby, 2014). De façon générale, les capacités que les élèves ingénieurs ont développées dans leurs approches des sciences de la nature et par les enseignements techniques ne les aident pas à se familiariser avec les démarches herméneutiques des SHS.

Puisque la technique ne saurait résoudre seule les problèmes qu'elle pose (Friedman, 1952), le temps est peut-être venu d'ouvrir les débats, dans les formations d'ingénieurs, sur les différentes formes de savoir. En effet, si un certain chemin a été parcouru depuis les années 1980, notamment sur les aspects de communication et de modélisation de la complexité, l'humilité sociale de l'expert reste à acquérir, tout comme la compréhension de la nécessité d'une multi-rationalité, pour affronter les défis contemporains.

\section{Conclusion}

En France, le modèle de formation des ingénieurs, mis en place sous l'Ancien Régime, est incontestablement un modèle élitiste, destiné à asseoir le pouvoir d'une classe dirigeante, notamment par une culture lettrée. Il s'agit de former l'honnête homme, au service de l'État, c'est à dire de l'intérêt général et du bien commun. C'est un premier mode de légitimation sociale des activités techniques des ingénieurs. Après la Révolution française, c'est sur la rationalité des sciences de la nature que s'est développé le modèle technocratique, se généralisant des ingénieurs d'État aux ingénieurs civils, destinés au monde industriel. Si ce modèle se voulait plus démocratique, il a néanmoins conservé les normes et les valeurs construites sur le sentiment d'exception que procure l'entrée dans des formations sélectives. Aujourd'hui, malgré la disparité des formations et en dépit de l'absence d'évidence nette entre les carrières des ingénieurs issus des différentes écoles, ces dernières restent fortement hiérarchisées, et en concurrence pour leur place dans cette hiérarchie. Autant que des contenus de savoirs et des savoir-faire, elles diffusent des savoir-être, caractéristiques des modes de socialisation qu'elles inculquent.

Dans la seconde moitié du XIXe siècle, la double naissance de la sociologie en France, à l'École des mines de Paris, par Frédéric Le Play et à l'université par Émile Durkheim, a pris place dans un contexte socialement, politiquement et religieusement bouleversé. La sociologie leplaysienne, basée sur l'étude du budget des ménages, et d'inspiration catholique, était conforme à l'ordre établi ; elle a servi d'appui au développement du rôle social de l'ingénieur. Intermédiaire entre le patronat et les ouvriers, ce dernier est ainsi devenu le garant de la paix sociale. À l'opposé, la sociologie laïque durkheimienne, rendant compte des constructions sociales des pouvoirs religieux, politiques et économiques, allait à l'encontre de la vision naturaliste et élitaire du catholicisme et des conservateurs de droite, auxquels étaient majoritairement ralliés les ingénieurs.

Par ailleurs, au tournant du $\mathrm{XX}^{\mathrm{e}}$ siècle, à l'École centrale de Paris, l'Organisation scientifique du travail (OST), promue par Le Chatelier, a permis de revisiter la nouvelle science industrielle comme une science de l'homme et du social, légitimée par le positivisme ambiant. Il est également possible que les ingénieurs généralistes aient eu besoin de l'OST pour asseoir leurs compétences, face à des ouvriers artisans qui avaient la maîtrise de leur métier, et peut-être aussi, face à des ingénieurs spécialisés qui avaient une maitrise technique. Mais quoi qu'il en soit, l'OST a rempli le rôle de pensée sociale pour les 
ingénieurs. La profondeur des liens, entre activité des ingénieurs (progrès technique) et préoccupations d'ordre social (amélioration du bien être humain et social), noués dans l'approche globalisante de la science industrielle, pourrait expliquer, autant le difficile positionnement des SHS dans de nombreuses formations d'ingénieurs, que les écarts de positionnement entre les différentes cultures technoscientifiques.

Par leur rôle utilitaire, centré sur la direction des hommes et le management des entreprises, les SHS ont à leur tour, suite aux humanités, acquis un rôle de légitimation d'une technocratie; ainsi, elles n'ont pas pu permettre aux ingénieurs d'exercer un discernement sur les choix de société qui étaient attendus d'eux après la seconde guerre mondiale. En effet, si face à la complexité et à l'incertitude grandissantes de nos sociétés, depuis les années 1980, les SHS ont des apports concrets à proposer, notamment par le développement des capacités à prendre en compte différents modes de rationalité, elles se heurtent encore souvent dans les formations d'ingénieurs à leurs spécificités épistémologiques, difficiles à appréhender pour des étudiants rompus aux modalités théoriques des sciences de la nature. Autrement dit, au delà des contenus des SHS, c'est leur mode de production des savoirs et leur mode de raisonnement qui soulèvent le plus de résistance dans les formations d'ingénieurs.

\section{BIBLIOGRAPHIE}

AERES (2010). Formation universitaire au métier d'ingénieur. AERES. Consulté à http://www.aeresevaluation.fr/Actualites/Actualites-de-l-agence/ETUDE-Formation-universitaire-au-metier-dingenieur

Bardel-Denonain, O. et Chaix, M. L. (1998). Éditorial. Recherche et Formation, 29, 5-10.

Belhoste, B. (2003). La formation d'une technocratie : l'École polytechnique et ses élèves de la Révolution au Second Empire. Paris : Belin.

Birck, F. (2006). De l'Institut électrotechnique de Nancy à l'École nationale supérieure d'électricité et de mécanique de Nancy. In F. Birck et A. Grelon (Dir.), Un siècle de formation des ingénieurs électriciens. Ancrage local et dynamiques européennes, l'exemple de Nancy (p. 23-88). Paris : MSH.

Blanckaert, C. (2006). La discipline en perspective Le système des sciences à l'heure du spécialisme (XIXe-XXe siècle). In J. Boutier, J. C. Passeron et J. Revel (Dir.), Qu'est-ce qu'une discipline ? (p. 117-147). Paris : EHESS.

Boltanski, L. (1981). America, America... Actes de la recherche en sciences sociales, 38(1), 19-41. doi : 10.3406/arss.1981.2116

Boure, R. (2007). Les sciences humaines et sociales en France : une approche historique. Cortil-Wodon : E.M.E. et Intercommunications.

Brancher, D. (1982). L'environnement et la formation des ingénieurs. Études. Paris : UNESCO. Consulté à http://unesdoc.unesco.org/images/0003/000391/039195fo.pdf 
Brucy, G. (1999). Formation ou culture, l'action des cadres et des ouvriers de la chimie CFTC-CFDT (1946-1971). Sociétés contemporaines, 35(1), 71-94. doi :10.3406/socco.1999.1740

Bruner, J. S. (2008). L'éducation, entrée dans la culture : les problèmes de l'école à la lumière de la psychologie culturelle. Paris : Retz (édition originale, 1996). (Ouvrage original publié en 1996 sous le titre The Culture of Education. New-York : Harward University Press).

Canepa, D., Folz, J. M. et Blazy, F. (2009). Mission d'étude sur l'avenir des corps d'ingénieurs de l'État. Paris : Rapport remis au Premier Ministre. Consulté à http://www.ladocumentationfrancaise.fr/ rapports-publics/094000145/

Cohen, Y. (2003). Fayol, un instituteur de l'ordre industriel. Entreprises et histoire, 34(3), 29-67. doi : 10.3917/eh.034.0029

Cohen, Y. (2011). Le « besoin de chefs » au début du XX siècle : Un tour d'horizon international entre business, politique et psychologie. Consulté à http://mtpf.mlab-innovation.net/fr/

Cohen, Y. et Baudoui, R. (1995). Gouverner le social, 1890-1945. In Y. Cohen et R. Baudouï (Dir.), Les chantiers de la paix sociale (p. 7-25). Paris : ENS Éditions.

Corbières, M. (2003). Le bizutage dans les écoles d'ingénieurs. Paris : L’Harmattan.

Crawford, S. (1984). Légitimité de l'autorité chez les ingénieurs. Culture Technique, 12, 246-253.

Dab, W. (2008). La formation des managers et ingénieurs en santé au travail. Douze propositions pour la développer. Rapport réalisé avec l'appui de la direction générale du travail et de l'Institut national de recherche et de sécurité. Rapport remis à Xavier Bertrand, ministre du travail, des relations sociales et de la solidarité et à Valérie Pécresse, ministre de l'enseignement supérieur et de la recherche. Paris. Consulté à http://www.ladocumentationfrancaise.fr/rapportspublics/084000404/

David, J. (2003). Régimes descriptifs du XIX ${ }^{e}$ siècle, le typique et le pittoresque dans l'enquête et le roman. In G. Blundo et J. P. Olivier de Sardan (Dir.), Pratiques de la description (p. 185-210). Paris : EHESS.

Day, C. (1991). Les Écoles d'Arts et Métiers : l'enseignement technique en France, XIX ${ }^{e}-X X^{e}$ siecles (J. P. Bardos, trad.). Paris : Belin (ouvrage original publié en 1987 sous le titre the Ecoles d'arts et metiers and the rise of french industrial engineering. Cambridge, Massachussetts : MIT Press).

Derouet, A. et Paye, S. (2010). Quand les ingénieurs débattent de leur formation non technique : socio-histoire d'un processus de repositionnement professionnel. Paris : Institut de recherches économiques et sociales (IRES), Confédération Générale des Cadres (CGC). Consulté à http:// archive.cfecgc.org/e_upload/pdf/etudeiresjuillet2010.pdf

Derouet, A. (2013). De l'honnête homme au manager ? La contribution des enseignements juridiques de l'École Centrale à la définition d'un ingénieur d'élite depuis 1829. Droit et société, 83 (1), 33-47.

Didier, C. (2008). Penser l'éthique des ingénieurs. Paris : PUF.

Dortier, J. F. (2006). Une histoire des sciences humaines. Auxerre : Éditions Sciences humaines Downey, G.L. et Lucena, J.C. (2004). Knowledge and Professional Identity in Engineering : CodeSwitching and the Metrics of Progress. History and Technology, 20(4), 393-420.

Dufour, A. (1998). Les enjeux de l'enseignement de la sociologie dans une École d'ingénieur. Analyse du curriculum de l'Institut supérieur d'agriculture Rhône-Alpes de 1968 à 1994 (Thèse de doctorat, Université Lumière Lyon 2, France). Consulté à http://theses.univ-lyon2.fr/documents/ lyon2/1998/adufour\#p=0\&a=top 
Duprez, J., Grelon, A. et Marry, C. (1991). Les ingénieurs des années 1990 : mutations professionnelles et identité sociale. Sociétés contemporaines, 6(1), 41-64. doi : 10.3406/ socco.1991.997

Eidelman, J. (1986). Science industrielle contre science pure : la professionnalisation de la recherche dans les années trente. In A. Grelon (Dir.), Les ingénieurs de la crise. Titre et profession entre les deux guerres (p. 113-133). Paris : EHESS.

Ellul, J. (1954). La technique ou l'enjeu du siècle. Paris : Armand Colin.

Friedman, G. (1952). Introduction : les conséquences sociales du progrès technique. Bulletin international des sciences sociales, IV(2) 251-272.

Fraysse, B. (1998). Professionnalisation et représentations socioprofessionnelles. Recherche et Formation, 29, 127-142.

Fraysse, B. (2000). La saisie des représentations pour comprendre la construction des identités. Revue des sciences de l'éducation, 26(3), 651. doi : 10.7202/000294ar

Fraysse, B. (Dir.). (2006). Professionnalisation des élèves-ingénieurs. Paris : L'Harmattan

Garçon, A. F. (2004). Entre l'État et l'usine : l'École des mines de Saint-Étienne au XIX ${ }^{e}$ siècle. Rennes : PUR.

Gervais, J. (2007). La réforme des cadres de l'action publique ou la fabrique d'un "nouveau " corps des Ponts et Chaussées Impératifs managériaux, logiques administratives et stratégies corporatistes (fin du XX siècle) (Thèse de doctorat, Université Lumière Lyon 2). Consulté à https://tel.archivesouvertes.fr/tel-00375142/

Giré, A., Béraud, A. et Déchamps, P. (2000). Les ingénieurs : identités en questions. Paris : L'Harmattan.

Godelier, É. (2009). La culture d'entreprise. Revue française de gestion, 192(2), 95-111.

Godin, B., Gingras, Y. et Bourneuf, E (1998). Les indicateurs de culture scientifique et technique. Rapport remis au ministère de l'industrie, du commerce et au ministère de la culture et des communications. Québec : Conseil de la science et de la technologie. Consulté à htp :// www4.bnquebec.ca/pgq/2005/3077070.pdf

Grelon, A. (1984). Les ingénieurs encore. Culture Technique, 12, 11-17.

Grelon, A. (1986). Introduction. L'évolution de la profession d'ingénieur en France dans les années 1930. In A. Grelon (Dir.), Les ingénieurs de la crise. Titre et profession entre les deux guerres (p. 7-32). Paris : EHESS.

Grelon, A. (1995). L'ingénieur catholique et son rôle social. In Y. Cohen et R. Baudouï (Dir.), Les chantiers de la paix sociale (p. 167-184). Paris : ENS Éditions.

Grelon, A. (1996). La naissance de l'enseignement supérieur industriel en France. Quaderns d'historia del engynieria, I, 40-60.

Grelon, A. et Marry, C. (1996). Entretien avec Bernard Decomps. Formation Emploi, 53, 49-63.

Grossetti, M. (1995). Science, industrie et territoire. Toulouse : Presses universitaires du Mirail. Consulté à https://halshs.archives-ouvertes.fr/halshs-00719731/PDF/Grossetti-Science-IndustrieTerritoire-1995.pdf

Heilbron, J. (2006). Naissance de la sociologie. Marseille : Agone

Heilbron, J., Lenoir, R. et Sapiro, G. (2004). Introduction. In J. Heilbron, R. Lenoir et G. Sapiro (Dir.), Pour une histoire des sciences sociales. Hommage à Pierre Bourdieu (p. 7-16). Paris : Fayard. 
Henry, O. (2004). De la sociologie comme technologie sociale. Actes de la recherche en sciences sociales, 153(3), 48-64. doi : 10.3917/arss.153.0048

Kalaora, B. et Savoye, A. (1992). Frédéric Le Play et les figures de l'ingénieur. Culture Technique, 26, 129-133.

Laot, F. (1999). La formation des adultes : histoire d'une utopie en acte, le complexe de Nancy. Paris :

L'Harmattan.

Lasserre, H. (1989). Le pouvoir de l'ingénieur. Paris : L'Harmattan.

Le Play, F., Savoye, A. et Audren, F. (2008). Naissance de l'ingénieur social : les ingénieurs des Mines et la science sociale au XIX ${ }^{e}$ siècle. Paris : Mines-ParisTech.

Lemaitre, D. (2003). La formation humaine des ingénieurs. Paris : PUF.

Lemaître, D. (2007). Entre savoirs et identités : le phénomène de « mimesis » dans la formation des ingénieurs. Les Sciences de l'éducation - Pour l'Ère nouvelle, 40(3), 11-29. doi : 10.3917/

lsdle.403.0011

Lenoir, R. (2004). Durkheim et la famille entre sociologie et politique. In J. Heilbron,

R. Lenoir et G. Sapiro (Dir.), Pour une histoire des sciences sociales. Hommage à Pierre Bourdieu (p.

27-45). Paris : Fayard.

Letté, M. (2004). Henry Le Chatelier (1850-1936) ou La science appliquée à l'industrie. Rennes : PUR.

Letté, M. (2010). Culture de la rationalisation chez les ingénieurs durant la seconde industrialisation. Communication présentée au Colloque, Les ingénieurs des Mines : cultures, pouvoirs, pratiques (p. 33-50). Paris : Institut de la gestion publique et du développement économique, Comité pour l'histoire économique et financière de la France, ENSMP, Paris, France. Consulté à https:// halshs.archives-ouvertes.fr/halshs-00541840/en/

Lourdel, N. (2005). Méthodes pédagogiques et représentation de la compréhension du développement durable : application à la formation des élèves-ingénieurs (Thèse de doctorat non publiée). École nationale supérieure des mines de Saint-Étienne, France.

Minguet, G. (2001). La formation des ingénieurs aux sciences sociales. Questions de légitimité. Sociologies pratiques, 5, 1-11.

Monnet, J. C. (2013). Un sociologue chez Renault : de Pierre Bourdieu à Carlos Ghosn, 1984-2005. Paris : Armand Colin.

Moutet, A. (1995). Sous le gouvernement de front populaire : problèmes humains de la rationalisation et action ouvrière. In Y. Cohen et R. Baudouï (Dir.), Les chantiers de la paix sociale ( $\mathrm{p}$. 287-310). Paris : ENS Éditions.

Moutet, A. (1997). Les logiques de l'entreprise : la rationalisation dans l'industrie française de l'entredeux-guerres. Paris : EHESS.

Picon, A. (1992). L'invention de l'ingénieur moderne : l'Ecole des ponts et chaussées, 1747-1851. Paris : Presses de l'École nationale des ponts et chaussées.

Picon, A. (2007). French Engineers and Social Thought, 18-20th Centuries : An Archeology of Technocratic Ideals. History and Technology, 23(3), 197-208. doi : 10.1080/07341510701300262

Picon, A. et Chatzis, K. (1992). La formation des ingénieurs français au siècle dernier. Débats, polémiques et conflits. L'orientation scolaire et professionnelle, 21(3), 227-243.

Pomian, K. (2010). Sciences humaines, sciences sociales : crise ou déclin ? Le Débat, 162(5), 19-35. doi : $10.3917 /$ deba.162.0019 
Remoussenard, P. (2006). La formation au métier d'ingénieur et ses limites à l'École nationale supérieure d'électricité et de mécanique de Nancy entre 1900 et 1960. In F. Birck et A. Grelon (Dir.), Un siècle de formation des ingénieurs électriciens. Ancrage local et dynamiques européenne, l'exemple de Nancy (p. 237-269). Paris : MSH.

Robert, J. L. (1986). Les syndicats d'ingénieurs et de techniciens et la protection du titre d'ingénieur (1919-1934). In A. Grelon (Dir.), Les ingénieurs de la crise. Titre et profession entre les deux guerres (p. 141-157). Paris : EHESS.

Roby, C. (2014). Place et fonction des SHS dans les écoles d'ingénieurs en France. État des lieux, enjeux et perspectives épistémologiques. Thèse de doctorat. Université Rennes 2.

Roquet, P. (2005). Émergences et représentations des modèles de formation des ingénieurs en France. In D. Poisson (Dir.), Complexité de la formation et formation à la complexité (p. 135-143). Paris : L'Harmattan. Consulté à cueep161.univ-lille1.fr/mcx/mcx/3b/3b-Roquet.doc

Sapiro, G. (2004). Défense et illustration de «l'honnête homme » Les hommes de lettres contre la sociologie. Actes de la recherche en sciences sociales, 153, 11-27.

Savoye, A. (1994). Les Débuts de la sociologie empirique : Études socio- historiques (1830-1930). Paris : Méridiens Klincksieck.

Savoye, A. (1995). Les enquêtes sur les budgets familiaux : la famille au microscope. In Y. Cohen et R. Baudouï (Dir.), Les chantiers de la paix sociale (p. 55-75). Paris : ENS Éditions.

Savoye, A. (2008). Introduction. In A. Savoye et F. Audren (Dir.), Frédéric Le play et ses élèves. Naissance de l'ingénieur social (p. 9-24). Paris : Mines-ParisTech.

Segrestin, D. (1996). Sociologie de l'entreprise. Paris : Armand Colin.

Serry, H. (2004). Saint Thomas sociologue ? Les enjeux cléricaux d'une sociologie catholique dans les années 1880-1920. Actes de la recherche en sciences sociales, 153, 28-40. doi : 10.3917/

arss.153.0028

Tanguy, L. (2001). Les promoteurs de la formation en entreprise (1945-1971). Travail et Emploi, 86, 27-47.

Thoenig, J. C. (1987). L’ère des technocrates : le cas des Ponts et chaussées. Paris : L'Harmattan.

Veltz, P. (2007). Faut-il sauver les grandes Écoles ? De la culture de la sélection à la culture de l'innovation . Paris : Les presses de Sciences po.

\section{NOTES}

1. Cette contribution tient compte des remarques faites par les relecteurs anonymes de l'article, qu'ils soient ici très sincèrement et chaleureusement remerciés, pour leurs conseils avisés.

2. 1850-1936, polytechnicien, chimiste, enseignant à l'École des mines, il obtient de Taylor le droit exclusif de traduire ses écrits en 1906 (Letté, 2004).

3. «Henry Le Chatelier délimite les contours de la science industrielle et la définit comme le modèle d'une organisation rationnelle de la recherche et développement dans l'entreprise, en vue de la rationalisation de la production, par le contrôle, la surveillance et l'optimisation de la fabrication courante, opérations elles-mêmes nécessairement standardisées (...) La rationalisation est dès lors entendue comme la définition des gestes et pratiques, inscrits dans le cadre d'une organisation et d'une hiérarchie clairement identifiée : la production est pensée par certaines personnes qui donnent des directives à d'autres personnes chargées d'une exécution scrupuleuse » (Letté, 2004, p. 134). 
4. Actes du séminaire CGE de Marseille 1er et 2 Octobre 2009.

5. Propos de Christiane Gillon, sociologue, intervenante en socioanalyse institutionnelle dans les centrales nucléaires, à une table-ronde organisée par Bardel-Denonain et Chaix (1998, p. 81).

\section{RÉSUMÉS}

Le positionnement des sciences humaines et sociales (SHS) dans les formations d'ingénieurs reste problématique. Par une approche socio-historique, cette contribution appréhende la place spécifique qu'ont tenue les ingénieurs dans la construction des savoirs sur l'humain et le social. Il apparaît que la recherche d'une légitimité sociale et technocratique pourrait expliquer la faible reconnaissance des SHS dans leur dimension critique, malgré leur intérêt pour l'étude des problèmes complexes.

The positioning of the humanities and social sciences (HSS) in the training of engineers remains problematic. By a socio-historic approach, this contribution deals with the specific place which held the engineers in the construction of the knowledges on the human beeings and the social. It appears that the search for a social and technocratic legitimacy could explain the low gratitude of the HSS in their critical dimension, in spite of their interest for the study of complex problems.

\section{INDEX}

Mots-clés : ingénieurs, SHS, légitimation, sociohistoire

Keywords : engineers, HSS, legitimacy, socio-historic approach

\section{AUTEUR}

\section{CATHERINE ROBY}

Université Bretagne Loire / Rennes 2, CREAD 